\title{
Seven Hundred Days of Startup: a Day in the Life of a Medical Student in Silicon Valley
}

\author{
Jonathan Y. Lee
}

Published online: 18 January 2014

(C) Academic Psychiatry 2014

Keywords Medical student $\cdot$ Startups $\cdot$ Innovation

Ever since I was a child, I had an odd obsession with changing rules to games in order to make them, at least to me, more fun: card games, board games, video games, and sports. No game was safe from my tinkering ways. It drove my brothers crazy. "Why can't we just play the game as it was meant to be played!?" they would shout. Looking back, I realize it came from a healthy disregard for the status quo. The status quo, like the original rules to games, is simply a fertile ground from which something better and something more effective can grow. As I aged, this inclination manifested itself in my academic and professional interests.

In medicine, the traditional path to innovation has been through research. As a medical student, there were countless opportunities to get involved in basic science, translational, and clinical research. However, having been part of the generation that witnessed the meteoric birth and rise of web startups like Google and Facebook, I was inspired to find ways to address problems in health care using web and mobile technology and startup tactics. Fortunately, we are in an era in which more and more clinicians and researchers are considering commercialization as a viable alternative to traditional research as a vehicle for change. One professor that had a profound influence on me noted his frustration after having to shut down his text message-based patient follow-up system, despite showing improved patient outcomes, because his grant had run out. Seeking other means of funding, he ended up incorporating the project and getting private investors. With a

J. Y. Lee $(\bowtie)$

University of Chicago, Chicago, IL, USA

e-mail: jonlee112@gmail.com compelling business case, commercial funding can sometimes be more readily available than research grants.

Startups, by nature, change quickly. Over the course of my 1 year at AgileMD, my days were drastically different. Instead of giving you a glimpse of just 1 day, I will highlight days 1 , 90, 500, and 700: validation, big dreams, great expectations, and goodbye and thanks.

\section{Day 1: Validation of an Idea}

The earliest days of a startup come before the startup is ever a company at all. It is just an idea with unrealized potential. The idea is to create a web/mobile platform that provides students and clinicians with access to clinical best practices in the form of interactive decision trees. Our goal is to validate the need for such a tool. To do this, we have spent weeks gathering data by talking to students, nurses, midlevel providers, physicians, other allied health professionals, insurance companies, and health tech companies. As an MD/MBA student in my fulltime business school year, I do this research outside of class responsibilities. Today, I have scheduled a phone call with a nurse practitioner at the student health center to ask her about the medical reference tools she favors when encountering a clinical question. After the call, I conduct a focus group with 4th year medical students to similarly detail their information gathering strategies. There is a definite art to doing "user research," and it is one that I have yet to master. How do you avoid asking leading questions? How do you unpack the deepest, most basic needs of a user? After these two research sessions, I compile the information and, with my teammates, spend several hours determining how to improve our product vision and pitch. The passion in the room is palpable, and I am excited to be working with this team.

Arguably, the most important thing you can do as an entrepreneur is to choose your teammates wisely. First are 
specialized skills. We needed two things in particular: knowledge of medicine and software engineers. No more. No less. Having too many cooks in the kitchen is a real problem. You do not need generalists, because anyone can do a generalist's work as long as they are passionate and humble. You do not want teammates who feel they are above desk assembly, lunch pick-up, or cold calls.

In a few days, we will be pitching our vision to a panel of judges at the University of Chicago, Booth School of Business New Venture Challenge, and the Chicago Biotech Consortium Startup Competition while also submitting an application to $\mathrm{Y}$ Combinator, a startup incubator program out in Silicon Valley that provides seed funding and mentorship to early stage startups. Our job is to convince all of them that we have identified a real need and are the right team to pull it off. In all of our nervousness, it would have been nice to know that we would go on to win first prize in both competitions and entry into Y Combinator.

\section{Day 90: a Small Startup with Big Dreams}

We have just finished the 3-month Y Combinator program. While doing a startup is often glamorized, especially in a movie about a particular social network, as involving lavish milestone celebrations, moments of genius, and romantic drama, the truth is that it is mostly a daily grind. With just a four-person team, my teammates and I each have a distinct role and a never-ending queue of tasks. Today, I wake up at 9:00 a.m., brush my teeth around the corner, and begin working at my desk $30 \mathrm{ft}$ away. While both sleeping and working at the same place are convenient, I find myself feeling a bit claustrophobic. Granted, I pause for meals and occasionally get some exercise in, but the first 6 months of Agile consist of close to $18 \mathrm{~h} /$ day, 7 days a week. It is hard to know when to stop working, as there is always something to be done. It feels as though the only limit to progress is the limited number of hours in a day. Ninety days into the company, we have a pretty solid idea of what our first launched product will look like, and so, we are doing less research now-more building. The initial pilot tool will contain interactive decision trees covering the management of 30 of the most common symptoms in internal medicine. For most of the day, I am using design tools to review, translate, and digitize clinical reasoning maps created by medical professors partnered with Agile. I also spend a lot of time on PubMed searching through guidelines to ensure that these algorithms represent current best practices. The particular tree I am trudging through is chest pain, which alone contains over 25,000 unique paths. The process is painstaking, but glimpsing into the way expert physicians reason is fascinating. I spend $2 \mathrm{~h}$ with one of my cofounders discussing the technical feasibility of a new interactive feature that, I believe, would enhance the usefulness of our tool. Being a medical student myself, I have an especially pertinent perspective on our potential user population. We plan to have a testable mockup of the feature ready 2 days from now. As such, I spend a couple of minutes emailing several of my old classmates for their availability for a feedback session. In these days, startups tend to emphasize a rapid iterative process to product building - prototype, test, prototype, test. At dinnertime, my teammates and I are relieved to take our minds off of work for a moment and enjoy an episode of our current favorite TV show. Shortly thereafter, we are back to work until late into the night. Eyes half closing, I finally retreat to bed. Did I brush my teeth? There are more important to things to worry about.

\section{Day 500: a Bigger Startup with Great Expectations}

The team is bigger now. We have raised a seed investment round and use some of the money to hire additional employees. We are up from 4 to 10 people. The days of worrying solely about my own projects are gone, as I am now directly managing several medical students who have taken a year off from school to work with us. Today is Monday, and we begin the day with a series of meetings. With a larger team, it becomes all the more important for the founding heads to have opportunities to discuss the overall goals and progress of the company's different arms. The four founders gather at 8:45 a.m. before any employees arrive and plan the upcoming week. At 9:45 a.m., the entire company gathers for the Monday meeting at 10 a.m., where the status of the last week's goals and plans for this week are discussed. At 10:30 a.m., I facilitate a final meeting with the medical team to distribute the week's tasks, while also allowing teammates to voice any questions, comments, or concerns. Managing people is often regarded as the most difficult aspect of organizations. It is partly project management and partly interpersonal relationship/family therapist. I still feel like a novice at this point, but it is incredibly fulfilling when the team is working effectively together.

From 11:00 a.m. to noon, everyone begins his/her day. I usually spend it getting organized, answering emails from the weekend, and setting a prioritized to-do list. At noon, a visitor arrives. One of the best things about Silicon Valley is that because you are surrounded by so many other startups, it is easy to find people dealing with similar obstacles. As such, we make it a point to invite other entrepreneurs over for lunch to share ideas and potentially plant the seed for future collaborations. Our visitor today is starting a health technology company that seeks to provide hospitals with HIPAA-compliant text messaging systems. We funnel out of the front door of the house that we call our "office" and enjoy a catered lunch from our favorite local Caribbean restaurant, "Coconuts" while sitting at the 10-ft picnic table on our patio. It is always a shot of adrenaline to listen to fellow entrepreneurs talk about their visions for health-care innovation. 
After lunch, the team returns to work. I begin my drive to a nearby academic medical center where I have scheduled a meeting with an $\mathrm{OB}-\mathrm{GYN}$ resident to discuss her progress on an abnormal uterine bleeding management decision tree. It also gives me a chance to get her feedback on a proposed feature that would enable users to take custom notes directly in our trees and protocols. After that meeting, I rush off to meet a nursing student and her professor who particularly enjoys teaching students using visual flow charts. Convinced that our system could be a great tool for her students, she agrees to pilot a set of educational trees with her students.

This is particularly a heavy day for meetings. A panel has been organized by the institution's technology interest group for faculty, students, local entrepreneurs, and investors interested in health technology. I am one of the panelists and take the opportunity to share not only the reasons I believe our startup can help improve the care of patients but also the reasons being part of a health tech startup has been an invaluable experience for me. Seeing the eager faces of students interested in startups reminds me of similar meetings I attended back in Chicago. After the panel, I head back down to Palo Alto. It is a 45-min drive, and I try to use the opportunity to catch up with family or friends over the phone. I return to the office around 9:00 p.m. and spend the next $3 \mathrm{~h}$ making progress on programming an experimental mobile application for a standardized patient discharge process to be part of a research project at another nearby academic medical center. Often, like today, one or two employees are still around, hard at work, and it is an inspiring scene to walk into at such a late hour. It reminds me of one of my favorite sayings: "If you want to go fast, go alone; if you want to go far, go together."

\section{Day 700: Goodbye and Thanks}

Today is officially my last day as an active founder and head of the medical team at Agile Diagnosis. Now, I turn my attention back to medical school, as I seek to complete my final year and pursue residency training. While I have learned a lot about growing a startup from just an idea, I have got a long road ahead of me when it comes to patient care.

I spend the morning wrapping up loose ends and finishing up a comprehensive document containing all the progress to date and next steps for the various active projects at Agile. I have made sure to hire exceptional medical students who will carry the torch from here.

At lunch, on that familiar picnic table in front of our house, I get a chance to tell everyone how fortunate I feel to have been able to call each of them a teammate for the last 2 years. I hold back tears as I reminisce about the 1st day moving to Silicon Valley and how far we have come. I came to Silicon Valley dreaming of changing the world. I leave this place knowing that with a good team, with good work ethic, and with good intentions, that is entirely possible...but not without a healthy disregard for the status quo.

\section{Implications for Academic Leaders}

- Startups are increasingly being viewed as a vehicle for health-care innovation that complements the traditional academic research infrastructure

- Students and clinicians can gain rare leadership experience, as they traverse the unique challenges of pushing a startup from idea to reality 